International Journal of Bifurcation and Chaos, Vol. 17, No. 12 (2007) 4431-4442

(c) World Scientific Publishing Company

\title{
ON THE STABILIZATION OF PERIODIC ORBITS FOR DISCRETE TIME CHAOTIC SYSTEMS BY USING SCALAR FEEDBACK
}

\author{
ÖMER MORGÜL \\ Bilkent University, Department of Electrical Engineering, \\ 06800, Ankara, Turkey \\ morgul@ee.bilkent.edu.tr
}

Received June 6, 2006; Revised October 20, 2006

\begin{abstract}
In this paper we consider the stabilization problem of unstable periodic orbits of discrete time chaotic systems by using a scalar input. We use a simple periodic delayed feedback law and present some stability results. These results show that all hyperbolic periodic orbits as well as some nonhyperbolic periodic orbits can be stabilized with the proposed method by using a scalar input, provided that some controllability or observability conditions are satisfied. The stability proofs also lead to the possible feedback gains which achieve stabilization. We will present some simulation results as well.
\end{abstract}

Keywords: Chaotic systems; chaos control; delayed feedback; Pyragas controller.

\section{Introduction}

The study of dynamical systems has attracted great attention in recent years due to its various potential applications, see e.g. [Chen \& Dong, 1998; Fradkov \& Pogromsky, 1998]. Among such various aspects, the study on feedback control in chaotic systems has received considerable interest after the seminal work of [Ott et al., 1990], where the term "controlling chaos" was introduced. Chaotic systems have many unstable periodic orbits embedded in their attractors and as is shown in [Ott et al., 1990], some of these orbits can be stabilized by using simple feedback laws. Since then, this subject has received great attention and many other feedback schemes to solve the same and related control problems have been proposed, see e.g. [Chen \& Dong, 1998; Fradkov \& Pogromsky, 1998; Solak et al., 2001]. Among such schemes, the delayed Feedback Control (DFC), first proposed by Pyragas in [Pyragas, 1992], and is also known as Pyragas scheme has received considerable interest due to its simplicity. Despite this simplicity, it was later shown that this scheme has certain inherent limitations, see e.g. [Ushio, 1996; Morgül, 2003a, 2005a]. A set of necessary and sufficient conditions to guarantee the local exponential stability of DFC have been given in [Morgül, 2003a, 2005a]. To overcome the limitations of DFC, various modifications of it have been proposed, see e.g. [Hino et al., 2002; Socolar et al., 1994; Kittel et al., 1995; Pyragas, 1995; Bleich \& Socolar, 1996; Schuster \& Stemmler, 1997; Nakajima \& Ueda, 1998]. Among these, the periodic feedback scheme proposed in [Schuster \& Stemmler, 1997] is quite interesting since it practically eliminates the limitations of DFC in one-dimensional case and various generalizations for multidimensional case are possible. Two such generalizations have been given in [Morgül, 2005b] and [Morgül, 2006].

In most of the works mentioned above, the control input has the same dimensionality as the original system. An interesting question was proposed by an anonymous reviewer during the publication process of [Morgül, 2005b] as to whether one can achieve the same objective with a control input with 
less dimensionality. A partial answer was given in [Morgül, 2005b], which works only in some limited cases. The present paper was inspired by the same question posed by the anonymous reviewer mentioned above. More precisely, we will consider the problem of stabilization of unstable periodic orbits of discrete time systems by using scalar control signals. Our approach is based on the stabilization scheme proposed in [Morgül, 2006].

This paper is organized as follows. In the next section, after introducing the basic notation, we will pose the problem considered in this paper. Then in Sec. 3 we will outline the basic stability results presented in [Morgül, 2006]. Then, in the following two sections we will give some solutions to the stated problem. After presenting some simulation results, finally we will present some concluding remarks.

\section{Problem Statement}

Let us consider the following discrete-time system

$$
x(k+1)=f(x(k)),
$$

where $k=1,2 \ldots$ is the discrete time index, $x \in \mathbf{R}^{n}$, $f: \mathbf{R}^{n} \rightarrow \mathbf{R}^{n}$ is an appropriate function, which is assumed to be differentiable wherever required. We assume that the system given by (1) possesses a $T$ periodic orbit characterized by the set

$$
\Sigma_{T}=\left\{x_{1}^{*}, x_{2}^{*}, \ldots, x_{T}^{*}\right\},
$$

i.e. for $x(1)=x_{1}^{*}$, the iterates of (1) yields $x(2)=$ $x_{2}^{*}, \ldots, x(T)=x_{T}^{*}, x(k)=x(k-T)$ for $k>T$.

Let $x(\cdot)$ be a solution of (1). To characterize the convergence of $x(\cdot)$ to $\Sigma_{T}$, we need a distance measure, which is defined as follows. For $x_{i}^{*}$, we will use circular notation, i.e. $x_{i}^{*}=x_{j}^{*}$ for $i=j(\bmod (T))$. Let us define the following indices $(j=1, \ldots, T)$ :

$$
d_{k}(j)=\sqrt{\sum_{i=0}^{T-1}\left\|x(k+i)-x_{i+j}^{*}\right\|^{2}},
$$

where $\|\cdot\|$ denotes any norm in $\mathbf{R}^{n}$. Without loss of generality, we will use standard Euclidean norm in the sequel. We then define the following distance measure

$$
d\left(x(k), \Sigma_{T}\right)=\min \left\{d_{k}(1), \ldots, d_{k}(T)\right\} .
$$

Clearly, if $x(1) \in \Sigma_{T}$, then $d\left(x(k), \Sigma_{T}\right)=0, \forall k$. Conversely if $d\left(x(k), \Sigma_{T}\right)=0$ for some $k_{0}$, then it remains 0 and $x(k) \in \Sigma_{T}$, for $k \geq k_{0}$. We will use $d\left(x(k), \Sigma_{T}\right)$ as a measure of convergence to the periodic solution given by $\Sigma_{T}$.
Let $x(\cdot)$ be a solution of (1) starting with $x(1)=x_{1}$. We say that $\Sigma_{T}$ is (locally) asymptotically stable if there exists an $\varepsilon>0$ such that for any $x(1) \in \mathbf{R}^{n}$ for which $d\left(x(1), \Sigma_{T}\right)<\varepsilon$ holds, we have $\lim _{k \rightarrow \infty} d\left(x(k), \Sigma_{T}\right)=0$. Moreover, if this decay is exponential, i.e. the following holds for some $M \geq 1$ and $0<\rho<1,(k>1)$ :

$$
d\left(x(k), \Sigma_{T}\right) \leq M \rho^{k} d\left(x(1), \Sigma_{T}\right),
$$

then we say that $\Sigma_{T}$ is (locally) exponentially stable.

To stabilize the periodic orbits of (1), let us apply the following control law:

$$
x(k+1)=f(x(k))+u(k),
$$

where $u(\cdot)$ is the control input. In classical DFC, the following feedback law is used $(k>T)$ :

$$
u(k)=K(x(k)-x(k-T)),
$$

where $K \in \mathbf{R}^{n \times n}$ is a constant gain matrix to be determined. It is known that the scheme given above has certain inherent limitations, see e.g. [Ushio, 1996]. For example, assume that $n=1$ and let $\Sigma_{1}=\left\{x_{1}^{*}\right\}$ be a period-1 orbit of (1) and set $a_{1}=f^{\prime}\left(x_{1}^{*}\right)$, where a prime denotes the derivative. It can be shown that $\Sigma_{1}$ can be stabilized with this scheme if $-3<a_{1}<1$ and cannot be stabilized if $a_{1}>1$, see [Ushio, 1996]. For $\Sigma_{T}$, let us set $a_{i}=f^{\prime}\left(x_{i}^{*}\right)$. It can be shown that $\Sigma_{T}$ cannot be stabilized with this scheme if $\prod_{i=1}^{T} a_{i}>1$, see e.g. [Ushio, 1996; Morgül, 2003a], and a similar condition can be generalized to the case $n>1$ [Hino et al., 2002]. A set of necessary and sufficient conditions to guarantee exponential stabilization for $n=1$ and $n \geq 1$ can be found in [Morgül, 2003a] and [Morgül, 2005a], respectively.

In (6) and (7), we have $u(k) \in \mathbf{R}^{n}$, i.e. the dimension of the control input is the same as that of the original system. A related question, as raised by one of the anonymous reviewers of [Morgül, 2005b] is to consider the following system:

$$
x(k+1)=f(x(k))+B u^{\prime}(k),
$$

where $B \in \mathbf{R}^{n}$ is the control vector and $u^{\prime}(k)$ is a scalar control input. Now the problem is, given the unstable periodic orbit $\Sigma_{T}$ of (1), to find appropriate control law $u^{\prime}(k)$ to stabilize $\Sigma_{T}$. In the sequel, we will pose two different versions of this problem and provide some solutions.

In most of the feedback schemes, the control input depends on the states, see e.g. (7). To show 
this dependence, let us consider the following form:

$$
u^{\prime}(k)=C^{T} u(k),
$$

where $C \in \mathbf{R}^{n}$ is the observation vector, here and in the sequel a superscript $T$ denotes the transpose and $u^{\prime}(k)$ is our main scalar control input. If we choose $u(k)$ according to the classical DFC scheme, we have

$$
u(k)=x(k)-x(k-T) .
$$

By comparing (8)-(10) with (6) and (7), we see that we have $K=B C^{T}$ in classical DFC. Using this form, we will pose two different problems:

Problem 1. Given $B \in \mathbf{R}^{n}$, find an appropriate $C \in \mathbf{R}^{n}$ and the control input $u(k)$ such that $\Sigma_{T}$ becomes stable.

Problem 2. Given $C \in \mathbf{R}^{n}$, find an appropriate $B \in \mathbf{R}^{n}$ and the control input $u(k)$ such that $\Sigma_{T}$ becomes stable.

Note that in Problem 1 , since $B$ is given, from the physical point of view the locations where we can apply the input has been given and we are required to find a scalar control input which would be applied to these locations to stabilize $\Sigma_{T}$. In Problem 2, the observation vector, hence the input signal to be used in stabilization, is given and we are required to find appropriate locations such that application of the control signal to these locations stabilize $\Sigma_{T}$. We note that while Problem 1 is related to the controllability of (8) and (9), Problem 2 is related to the observability of the same system. As a reminder, assume that a matrix $A \in \mathbf{R}^{\mathbf{n} \times \mathbf{n}}$ and two vectors $B, C \in \mathbf{R}^{\mathbf{n}}$ are given. Then, the pair $(A, B)$ is called controllable if

$$
\operatorname{rank}\left(B \quad A B \quad A^{2} B \cdots A^{n-1} B\right)=n,
$$

whereas the pair $(C, A)$ is called observable if the pair $\left(A^{T}, C\right)$ is controllable, see e.g. [Kailath, 1980].

\section{Basic Stability Results}

Let us assume that a periodic orbit $\Sigma_{T}$ for the system (1) is given and we consider the feedback control system given by (6). To stabilize $\Sigma_{T}$, various control schemes were proposed in the literature, see e.g. [Morgül, 2005b, 2006]. The scheme which will be used in the present paper is based on the latter, and is called as Double Period DFC (DPDFC). Let us assume that $T=m \geq 1$. According to this scheme, the control law is given as:

$$
u(k)=\epsilon(k)(x(k-m+1)-x(k-2 m+1)),
$$

where $\epsilon(k)$ is given as:

$$
\epsilon(k)=\left\{\begin{array}{ll}
K & k(\bmod 2 m)=0 \\
0 & k(\bmod 2 m) \neq 0
\end{array} .\right.
$$

It can easily be shown that for the case $m=1$, the scheme given above reduces to the scheme proposed in [Schuster \& Stemmler, 1997]. However, for the case $m>1$, the scheme proposed above and the one proposed in [Schuster \& Stemmler, 1997] are quite different. To see the relation between the control laws given in [Schuster \& Stemmler, 1997] and the one given above, let $\Sigma_{m}$ given by (2) be a period $m$ solution of (1). Let us define the $m$-iterate map $F$ as $F=f^{m}$. Clearly period $m$ orbits of $f$ are equivalent to period-1 orbits of $F$, i.e. $F\left(x_{i}^{*}\right)=x_{i}^{*}$, $i=1,2, \ldots, m$. Let us set

$$
z(j)=x((j-1) m+1), \quad j=1,2, \ldots .
$$

If $j$ is odd, by using (12) and (13) in (6), we obtain:

$$
x(j m+1)=f(x(j m))=f^{m}(x((j-1) m+1)),
$$

which is the same as

$$
z(j+1)=F(z(j)) .
$$

On the other hand, if $j$ is even, similarly we obtain:

$$
\begin{aligned}
x(j m+1)= & f^{m}(x((j-1) m+1)) \\
& +K(x((j-1) m+1) \\
& -x((j-2) m+1)),
\end{aligned}
$$

which is the same as

$$
z(j+1)=F(z(j))+K(z(j)-z(j-1)) .
$$

By combining (16) and (18), we see that in terms of the variable $z$ as defined in (14), we have the following dynamics:

$$
z(j+1)=F(z(j))+u(j),
$$

where $u(j)$ is given by:

$$
u(j)=\epsilon(j)(z(j)-z(j-1)),
$$

and $\epsilon(\cdot)$ is given by (13). We note that (19) and (20) are similar to the scheme proposed in [Schuster \& Stemmler, 1997] for the case $m=1$ in the variable $z$ defined in (14).

As explained above, any periodic point $x_{i}^{*}$ of $\Sigma_{m}$ is a period-1 orbit of the $m$-iterate map $F=$ $f^{m}$. In other words, we have $F\left(x_{i}^{*}\right)=x_{i}^{*}$. Let us define the error $e$ as $e(k)=z(k)-x_{i}^{*}$. By using 
(13), (19), (20), after linearization and considering only the first order terms, we obtain:

$$
\begin{aligned}
& e(2)=J_{i} e(1), \\
e(3)= & \left(J_{i}+K\right) e(2)-K e(1) \\
= & \left(J_{i}^{2}+K\left(J_{i}-I\right)\right) e(1),
\end{aligned}
$$

where $I$ is the identity matrix and $J_{i}$ is the Jacobian of $F$ evaluated at $x_{i}^{*}$, i.e.

$$
J_{i}=\left.\frac{\partial F}{\partial z}\right|_{z=x_{i}^{*}} .
$$

By using the mathematical induction, and repeating the procedure given above, we obtain:

$$
e(2 k+1)=\left(J_{i}^{2}+K\left(J_{i}-I\right)\right)^{k} e(1),
$$

for any $k$. The proof of this fact is omitted here, since it can easily be done by linearization and a similar analysis given above. From (24), it follows easily that the linearized error dynamics is locally exponentially stable if and only if the matrix $A_{i}$ given below

$$
A_{i}=J_{i}^{2}+K\left(J_{i}-I\right),
$$

is stable, i.e. all of the eigenvalues of $A_{i}$ are inside the unit disc. For the computation of $J_{i}$ in terms of $f$, let us define the matrices $D_{j}$ as Jacobian matrices of $f$ at periodic points of $\Sigma_{m}$, i.e.

$$
D_{j}=\left.\frac{\partial f}{\partial x}\right|_{x=x_{j}^{*}}, \quad j=1, \ldots, m .
$$

Since $F=f^{m}$, by using chain rule we obtain the following relation

$$
J_{i}=D_{i} D_{i+1}, \ldots, D_{i+m-1},
$$

see e.g. [Devaney, 1987; Alligood et al., 1997]. Note that here we employ the circular notation, i.e. $D_{i}=$ $D_{j}$ if $i=j(\bmod m)$.

Remark 1. We note that since any periodic point $x_{j}^{*}$ is different from others on the periodic orbit given by $\Sigma_{m}$, the resulting Jacobian matrices $D_{j}$ given by (26) are also different at different points on $\Sigma_{m}$, i.e. $D_{i} \neq D_{j}$ for $i \neq j$, in general. Hence, as a result the same holds for $J_{i}$ given by (27), see e.g. p. 71 of [Alligood et al., 1997]. However, since $\Sigma_{m}$ is a period $m$ orbit of (1), the set of eigenvalues of $J_{i}$ are the same for any $i=1,2, \ldots, m$, see e.g. [Devaney, 1987], or Lemma A.2, p. 558 of [Alligood et al., 1997]. This is basically due to the circular permutation given in (27), see e.g. [Alligood et al., 1997]. This shows that, in the uncontrolled case (i.e. when $K=0$ ), the stability of $\Sigma_{m}$ can be determined by any matrices $J_{i}$, see e.g. [Devaney, 1987; Alligood et al., 1997]. For the controlled case, since $J_{i} \neq J_{j}$ for $i \neq j$, in general, for a given control gain matrix $K$, we also have $A_{i} \neq A_{j}$ for $i \neq j$, in general, see (25). As a result, the stability properties of these matrices may differ, since they do not necessarily obey the circular permutation rule, see (25) and (27). We will demonstrate this point in the simulation section, see Remark 7.

Now consider the fixed points of $F$, which can be given as $\Sigma_{1}^{i}=\left\{x_{i}^{*}\right\}$. Since

$$
\Sigma_{m}=\Sigma_{1}^{1} \cup \Sigma_{1}^{2} \cdots \cup \Sigma_{1}^{m}
$$

for the stability of $\Sigma_{m}$, we require that at least one of the matrices $A_{i}$ be stable. In this case, if the initial condition $x(0)$ is sufficiently close to $x_{i}^{*}$ and if we apply the control law given above, the derivations given above shows that $d\left(x(k), \Sigma_{m}\right) \rightarrow 0$ as $k \rightarrow \infty$.

Recall that a matrix is called stable if all of its eigenvalues are inside the unit disc, unstable if at least one of its eigenvalues is outside the unit disc, and marginally stable if at least one of its eigenvalues is on the unit disc while the rest of its eigenvalues are inside the unit disc. We can summarize these results as follows.

Theorem 1. Let a period $m$ orbit of (1) be given as $\Sigma_{m}=\left\{x_{1}^{*}, \ldots, x_{m}^{*}\right\}$ and let us define the matrices $D_{i}$ and $J_{i}$ as given in (26) and (27), respectively. The DPDFC scheme given by (6), (12) and (13) is

(i) locally exponentially stable if and only if at least one of the matrices $A_{i}$ given by (25) is stable,

(ii) not stable if all of the matrices $A_{i}$ are unstable.

(iii) This analysis is inconclusive if all of the matrices $A_{i}$ are marginally stable.

Proof. Note that the local exponential stability is equivalent to the stability of the linearized system, see e.g. [Khalil, 2002]. The proof of the theorem then easily follows from standard Lyapunov stability arguments.

Now let us consider the problem of finding an appropriate gain matrix $K$ for the stabilization of $\Sigma_{m}$. Although for a given $K$ the stability properties of $A_{i}$ may be different, the solvability of this problem depends only on the eigenvalues of $J_{i}$. Also note that the eigenvalues of $J_{i}$ are the same for all $i$, see Remark 1. The solution of the problem of 
finding appropriate gain $K$ is given in the following Corollary.

Corollary 1. Let a period $m$ orbit of (1) be given as $\Sigma_{m}=\left\{x_{1}^{*}, \ldots, x_{m}^{*}\right\}$ and let us define the matrices $D_{i}$ and $J_{i}$ as given in (26) and (27), respectively. There exists a gain matrix $K$ such that the DPDFC scheme given by (6), (12) and (13) is locally exponentially stable if and only if $\lambda=1$ is not an eigenvalue of $J_{i}$ for any (hence for all) $i=1,2, \ldots, m$.

Proof. Assume that $\lambda=1$ is not an eigenvalue of $J_{i}$. Hence, $J_{i}-I$ is invertible. Let $X \in \mathbf{R}^{n \times n}$ denote an arbitrary stable matrix. Let us choose $K$ as

$$
K=\left(-J_{i}^{2}+X\right)\left(J_{i}-I\right)^{-1}
$$

Substituting (29) in (25) we obtain $A_{i}=X$; hence with this choice $A_{i}$ becomes a stable matrix.

Now assume that $\lambda=1$ is an eigenvalue of $J_{i}$. Let $\phi \in \mathbf{R}^{n}$ be the corresponding eigenvector of $J_{i}$. By using the fact $J_{i} \phi=\phi$, we obtain

$$
A_{i} \phi=J_{i}^{2} \phi+K\left(J_{i}-I\right) \phi=J_{i}^{2} \phi=\phi .
$$

Hence $\lambda=1$ is then an eigenvalue of $A_{i}$, independent of $K$. Since the eigenvalues of $J_{i}$ are the same, see Remark 1, it follows that independent of $K$, none of the matrices $A_{i}$ is stable. Therefore, by Theorem 1 , there cannot be a $K$ such that the DPDFC is locally exponentially stable.

For more details of this subject, see [Morgül, 2006].

\section{A Solution for Problem 1}

In this section, we will consider the system given by (8) and (9) where $u(k)$ is given by (12) and (13). By using the latter equation in the former and comparing with (6) and (7), we see that in this case we have $K=B C^{T}$. Since we assume that $B$ is given, our aim is to find an appropriate $C$ such that the matrix $A_{i}$ given by (25) becomes stable for some $i$. A solution can be found by using the standard controllability theory, which is provided below.

Theorem 2. Let a period $m$ orbit of (1) be given as $\Sigma_{m}=\left\{x_{1}^{*}, \ldots, x_{m}^{*}\right\}$ and let us define the matrices $D_{i}$ and $J_{i}$ as given in (26), (27), respectively, and consider the DPDFC scheme given by (6), (12) and (13). Then, there exists a vector $C \in \mathbf{R}^{n}$ such that the DPDFC scheme is locally exponentially stable if the following conditions hold:

(i) The pair $\left(J_{i}^{2}, B\right)$ is controllable at least for one $i=1,2, \ldots, m$.

(ii) $\lambda=1$ is not an eigenvalue of $J_{i}$ for any (hence for all) $i=1,2, \ldots, m$.

Proof. It is well known from linear system theory that if the pair $\left(J_{i}^{2}, B\right)$ is controllable, then there exists a vector $F_{i} \in \mathbf{R}^{n}$ such that the matrix $L_{i}=$ $J_{i}^{2}+B F_{i}^{T}$ is stable; moreover, the eigenvalues of $L_{i}$ can be assigned arbitrarily by an appropriate choice of $F_{i}$, see e.g. [Kailath, 1980]. By comparing $L_{i}$ with $A_{i}$ given by (25) and noting that $K=B C^{T}$, we see that we have $F_{i}^{T}=C^{T}\left(J_{i}-I\right)$. If $\lambda=1$ is not an eigenvalue of $J_{i}$, then $\left(J_{i}-I\right)$ is invertible and we can find the required vector $C$ as:

$$
C^{T}=F_{i}^{T}\left(J_{i}-I\right)^{-1} .
$$

Remark 2. An algorithm to compute $F_{i}$ will be provided in the following sections.

Remark 3. The conditions given in Theorem 2 become both necessary and sufficient for local exponential stability when the term "controllable" is replaced by "stabilizable", which guarantees the existence of a vector $F_{i}$ such that the matrix $L_{i}$ given above becomes stable; however in this case some of the eigenvalues of $L_{i}$, although stable, may become fixed and may not be assigned arbitrarily, see e.g. [Kailath, 1980]. Necessity of this condition is also obvious since we have $K=B C^{T}$ in (25). For the necessity of the condition (ii), let us use contradiction. Assume that $\lambda=1$ is an eigenvalue of $J_{i}$ for some (hence for all) $i=1,2, \ldots, m$. Let $\phi_{i} \in \mathbf{R}^{n}$ be the corresponding eigenvector. Then we have:

$$
A_{i} \phi_{i}=\left(J_{i}^{2}+B C^{T}\left(J_{i}-I\right)\right) \phi_{i}=J_{i}^{2} \phi_{i}=\phi_{i} .
$$

Hence $\lambda=1$ is an eigenvalue of $A_{i}$ for all $i=1,2, \ldots, m$. Therefore, by Theorem 2 , DPDFC scheme cannot be exponentially stable. Sufficiency is obvious from Theorem 2.

\section{A Solution for Problem 2}

In this section, we will consider the system given by (8) and (9) where $u(k)$ is given by (12) and (13). By using the latter equation in the former and comparing with (6) and (7), we see that in this case we have $K=B C^{T}$. Since we assume that $C$ is given, our aim is to find an appropriate $B$ such that the matrix $A_{i}$ given by (25) becomes stable for some $i$. A solution 
can be found by using the standard observability theory, which is provided below.

Theorem 3. Let a period $m$ orbit of (1) be given as $\Sigma_{m}=\left\{x_{1}^{*}, \ldots, x_{m}^{*}\right\}$ and let us define the matrices $D_{i}$ and $J_{i}$ as given in (26) and (27), respectively, and consider the DPDFC scheme given by (6), (12) and (13). Then, there exists a vector $B \in \mathbf{R}^{n}$ such that the DPDFC scheme is locally exponentially stable if the following conditions hold:

(i) The pair $\left(C, J_{i}^{2}\right)$ is observable at least for one $i=1,2, \ldots, m$.

(ii) $\lambda=1$ is not an eigenvalue of $J_{i}$ for any (hence for all) $i=1,2, \ldots, m$.

Proof. Proof is similar to that of Theorem 2. It is well known from linear system theory that if the pair $\left(C, J_{i}^{2}\right)$ is observable, then there exists a vector $F_{i} \in \mathbf{R}^{n}$ such that the matrix $L_{i}^{\prime}=J_{i}^{2}+F_{i} C^{T}$ is stable; moreover, the eigenvalues of $L_{i}^{\prime}$ can be assigned arbitrarily by an appropriate choice of $F_{i}$, see e.g. [Kailath, 1980]. If $\lambda=1$ is not an eigenvalue of $J_{i}$, then $\left(J_{i}-I\right)$ is invertible and hence the eigenvalues of $L_{i}=\left(J_{i}-I\right)^{-1} L_{i}^{\prime}\left(J_{i}-I\right)$ are the same as $L_{i}^{\prime}$. Therefore, we have:

$$
\begin{aligned}
L_{i}= & \left(J_{i}-I\right)^{-1} J_{i}^{2}\left(J_{i}-I\right) \\
& +\left(J_{i}-I\right)^{-1} F_{i} C^{T}\left(J_{i}-I\right) \\
= & J_{i}^{2}+\left(J_{i}-I\right)^{-1} F_{i} C^{T}\left(J_{i}-I\right),
\end{aligned}
$$

since we have $J_{i}^{2}\left(J_{i}-I\right)=\left(J_{i}-I\right) J_{i}^{2}$. By comparing (33) with $A_{i}$ given by (25), we see that

$$
B=\left(J_{i}-I\right)^{-1} F_{i} .
$$

Remark 4. An algorithm to compute $F_{i}$ will be provided in the following sections.

Remark 5. The conditions given in Theorem 3 become both necessary and sufficient for local exponential stability when the term "observable" is replaced by "detectable", which guarantees the existence of a vector $F_{i}$ such that the matrix $L_{i}^{\prime}$ given above becomes stable; however, in this case some of the eigenvalues of $L_{i}^{\prime}$, although stable, may become fixed and may not be assigned arbitrarily, see e.g. [Kailath, 1980]. The rest of the proof of this fact is the same as given in Remark 3.

Remark 6 . We note that the period $m$ orbit given by $\Sigma_{m}$ is called hyperbolic if any (hence all) of the Jacobians $J_{i}$ does not have an eigenvalue on the unit circle, see e.g. [Devaney, 1987]. According to our results, any hyperbolic, as well as some nonhyperbolic periodic orbits can be stabilized by our method, provided that the mentioned controllability or observability conditions are satisfied. For more details, see e.g. [Morgül, 2005b, 2006].

Before we proceed, we will first give a simple algorithm to obtain appropriate vectors $F_{i}$ used in previous sections, see (31) and (34). The technique we use is a well-known transformation technique used in [Morgül, 2003b]. Let us assume that the matrix $A=J_{i}^{2}$ and the vector $B$ be given. Furthermore, let us assume that the pair $(A, B)$ is controllable, i.e. (11) holds. This means that the following matrix is invertible:

$$
Q_{c}=\left(\begin{array}{lll}
A^{n-1} B & A^{n-2} B \cdots A B & B
\end{array}\right) .
$$

Let $p(\lambda)$ be the characteristic polynomial of $A$ given as:

$$
\begin{aligned}
p(\lambda) & =\operatorname{det}(\lambda I-A) \\
& =\lambda^{n}+\alpha_{1} \lambda^{n-1}+\cdots+\alpha_{n-1} \lambda+\alpha_{n} .
\end{aligned}
$$

Now, let us define the vectors $u_{1}=\left(\begin{array}{llll}1 & \alpha_{1} \cdots \alpha_{n-1}\end{array}\right)^{T}$, $u_{2}=\left(\begin{array}{lllll}0 & 1 & \alpha_{1} & \cdots & \alpha_{n-2}\end{array}\right)^{T}, \ldots, u_{n}=\left(\begin{array}{llll}0 & 0 & \cdots & 1\end{array}\right)^{T}$, and define the matrices $U=\left(u_{1} u_{2} \cdots u_{n}\right), R=\left(Q_{c} U\right)^{-1}$. Now consider the matrix $A_{c}=A+B K^{T}$. Obviously $A_{c}$ has the same eigenvalues as $R A_{c} R^{-1}$, i.e.

$$
R A_{c} R^{-1}=R A R^{-1}+R B K^{T} R^{-1},
$$

where after simple calculations one easily obtains

$$
\begin{gathered}
R A R^{-1}=\left(\begin{array}{ccccc}
0 & 1 & 0 & \cdots & 0 \\
0 & 0 & 1 & \cdots & 0 \\
& & \vdots & & \\
0 & 0 & 0 & \cdots & 1 \\
-\alpha_{n} & -\alpha_{n-1} & -\alpha_{n-2} & \cdots & -\alpha_{1}
\end{array}\right), \\
R B=\left(\begin{array}{c}
0 \\
0 \\
0 \\
0 \\
1
\end{array}\right) .
\end{gathered}
$$

We note that the form given above is known as controllable canonical form in control theory, see e.g. [Kailath, 1980]. We note that the form given above is also called Brunowsky canonical form and has an important application in chaos synchronization, see 
e.g. [Morgül \& Solak, 1996, 1997]. Let $p_{s}(\lambda)$ be an arbitrary but stable polynomial given as

$$
p_{s}(\lambda)=\lambda^{n}+a_{1} \lambda^{n-1}+\cdots+a_{n-1} \lambda+a_{n} .
$$

Then, by comparing (37) and (38), it follows that an appropriate choice of $K$ to make the matrix $A_{c}$ stable is:

$K^{T}=\left(\begin{array}{ll}\alpha_{n}-a_{n} & \alpha_{n-1}-a_{n-1} \cdots \alpha_{1}-a_{1}\end{array}\right) R$.

Since in our case we try to make the matrix $J_{i}^{2}+$ $B C^{T}\left(J_{i}-I\right)$ stable, assuming that $B$ is given, we have $K^{T}=C^{T}\left(J_{i}-I\right)$. Hence, by using (40), it follows that an appropriate choice for $C$ is given as:

$$
\begin{aligned}
C^{T}= & \left(\alpha_{n}-a_{n} \quad \alpha_{n-1}-a_{n-1} \cdots \alpha_{1}-a_{1}\right) \\
& \times R\left(J_{i}-I\right)^{-1} .
\end{aligned}
$$

On the other hand, if $C$ is given, by using Theorem 3 and following the same methodology, an appropriate $B$ can be found as:

$$
\begin{aligned}
B= & \left(J_{i}-I\right)^{-1} R^{T} \\
& \times\left(\alpha_{n}-a_{n} \quad \alpha_{n-1}-a_{n-1} \cdots \alpha_{1}-a_{1}\right)^{T} .
\end{aligned}
$$

\section{A Simple Implementation}

For the simulations, we will use a simple modification of the DPDFC algorithm given above. This modification, which is also used in [Morgül, 2003a, 2005a, 2005b], does not change the generality of the results. Note that the DPDFC scheme given by (12) and (13) achieves only local stabilization, i.e. it achieves stabilization only when the solutions of (6) are sufficiently close to the periodic orbit in certain sense. Hence, from implementation point of view, it is reasonable to apply DPDFC only when the solutions are sufficiently close to $\Sigma_{m}$. Let $\rho(k)$ denote an appropriate function which measures the closeness of trajectories to $\Sigma_{m}$, and let $\epsilon_{m}>0$ denote a constant related to the size of the domain of attraction of $\Sigma_{m}$. A reasonable implementation of DPDFC, which we will use in our simulations, is given as follows:

$$
\begin{gathered}
x(k+1)=f(x(k))+u(k), \\
u(k)=\epsilon(k)(x(k-m+1)-x(k-2 m+1)), \\
\epsilon(k)= \begin{cases}K & k(\bmod 2 m)=0 \text { and } \rho(k)<\epsilon_{m} \\
0 & \text { otherwise }\end{cases}
\end{gathered}
$$

Note that in our case we have $K=B C^{T}$. Since the solutions of (43) are chaotic for $u=0$, eventually the trajectories of the uncontrolled system will enter into the domain of attraction of $\Sigma_{m}$, i.e. $\rho(k)<\epsilon_{m}$ will be satisfied for some $k$, and hence afterwards the DPDFC given by (43)-(45) will be effective. Also, with this modification DPDFC will achieve stabilization for any initial condition in the domain of attraction of the chaotic attractor of (12). Obviously, for higher order periodic orbits, the time required till the trajectories enter into the domain of attraction of $\Sigma_{m}$ will be larger. Although the choice of $\rho(k)$ may vary, a reasonable selection, which we will use in our simulations, is the distance measure $d\left(x(k), \Sigma_{m}\right)$ given in (4).

\section{Simulation Results}

We will present two sets of simulations. The first set is related to the Problem 1. Let us consider the well-known Hénon system given below:

$$
\begin{gathered}
x_{1}(k+1)=1+x_{2}(k)-a x_{1}(k)^{2}, \\
x_{2}(k+1)=b x_{1}(k),
\end{gathered}
$$

where the parameters are chosen as $a=1.4, b=0.3$, for which the system exhibits chaotic behavior. In particular, for this parameter set, the system given above has an unstable period 2 orbit $\Sigma_{2}=\left\{w_{1}^{*}, w_{2}^{*}\right\}$ given as:

$$
\begin{aligned}
& w_{1}^{*}=\left(\begin{array}{r}
0.975800051 \\
-0.142740015
\end{array}\right), \\
& w_{2}^{*}=\left(\begin{array}{r}
-0.475800051 \\
0.292740015
\end{array}\right) .
\end{aligned}
$$

First, let us assume that the control vector is given as $B=\left(\begin{array}{ll}1 & 0\end{array}\right)^{T}$, i.e. the control input is only applied to (46). The Jacobian matrices $D_{i}$ and $J_{i}$, $i=1,2$, given by (26) and (27) can be computed easily as:

$$
\begin{gathered}
D_{1}=\left(\begin{array}{ll}
-2.7322401428 & 1 \\
0.3 & 0
\end{array}\right), \\
D_{2}=\left(\begin{array}{ll}
1.3322401428 & 1 \\
0.3 & 0
\end{array}\right), \\
J_{1}=D_{1} D_{2}=\left(\begin{array}{cc}
-3.3898 & -2.7322 \\
0.3997 & 0.3
\end{array}\right), \\
J_{2}=D_{2} D_{1}=\left(\begin{array}{ll}
-3.3898 & 1.3322 \\
-0.8196 & 0.3
\end{array}\right) .
\end{gathered}
$$

Hence the characteristic polynomial $p(\lambda)=$ $\operatorname{det}\left(\lambda I-J_{1}^{2}\right)$ can be computed as $p(\lambda)=\lambda^{2}-$ $9.0606 \lambda+0.0081$. Therefore, we have $\alpha_{1}=-9.0606$, $\alpha_{2}=0.0081$, see (36). It can easily be shown 
that the pair $\left(J_{1}^{2}, B\right)$ is controllable. The required transformation matrix $R$ given before (37) can be found as:

$$
R=\left(\begin{array}{cc}
0 & -0.831 \\
1 & 0.8247
\end{array}\right)
$$

By choosing the stable polynomial $p_{s}$ given by (39) as $p_{s}(\lambda)=\lambda^{2}$, (i.e. $a_{1}=a_{2}=0$ ) and using these in (41), we obtain:

$$
C^{T}=(2.2595 \quad 1.8651) .
$$

We simulated the DPDFC scheme given by (43)(45) for the Hénon system given by (46)-(49) for the parameters and gain vectors given above under various initial conditions and obtained satisfactory results. Note that we have $K=B C^{T}$ where $B^{T}=$ (1 0 ) and $C$ is given by (52). By using these in (46) and (47), the controlled system becomes:

$$
\begin{gathered}
x_{1}(k+1)=1+x_{2}(k)-a x_{1}(k)^{2}+u(k), \\
x_{2}(k+1)=b x_{1}(k),
\end{gathered}
$$

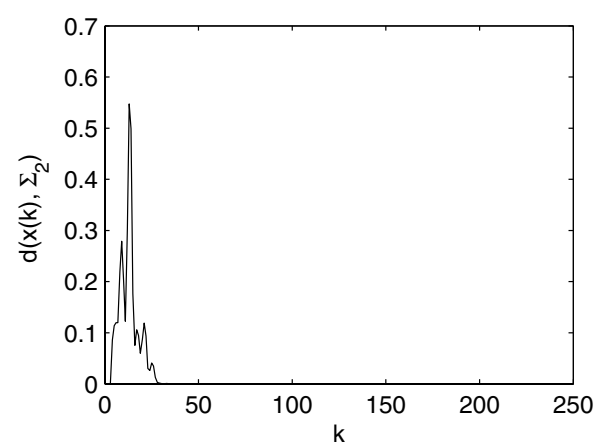

(a)

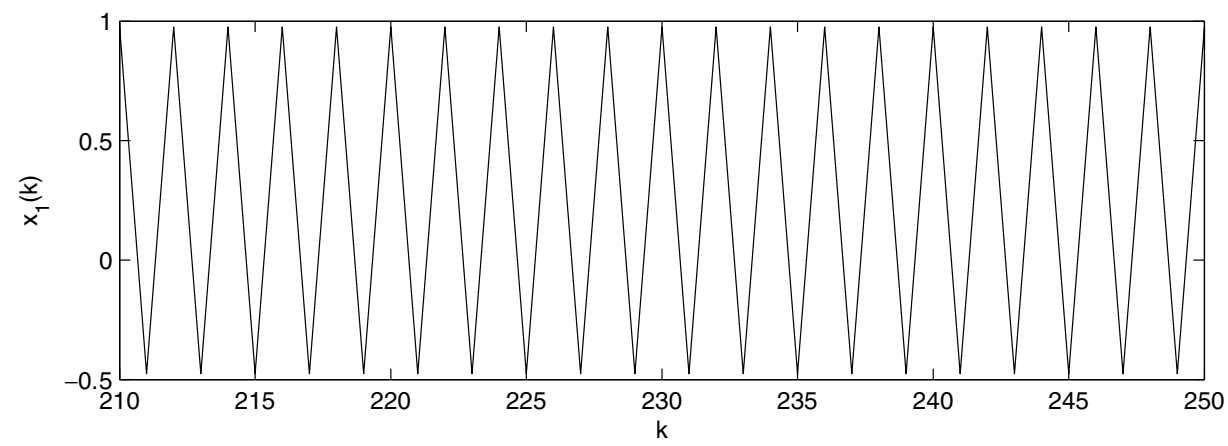

(c)

Fig. 1. DPDFC applied to Hénon map, $B=\left(\begin{array}{ll}1 & 0\end{array}\right)^{T}$, (a) $d\left(x(k), \Sigma_{2}\right)$ versus $k$, (b) $u(k)$ versus $k$, (c) $x_{1}(k)$ versus $k$ for $210 \leq k \leq 250$. where $u(k)$ is given by (44) and $\epsilon(k)$ is given by

$\epsilon(k)=\left\{\begin{array}{ll}C^{T} & k(\bmod 2 m)=0 \text { and } d\left(x(k), \Sigma_{m}\right)<\epsilon_{m} \\ 0 & \text { otherwise }\end{array}\right.$,

and $x=\left(\begin{array}{ll}x_{1} & x_{2}\end{array}\right)^{T}$. A particular simulation result for $x_{1}(1)=-0.32, x_{2}(1)=0.11$ and $\epsilon_{m}=0.6$ is given in Fig. 1. Figure $1(\mathrm{a})$ shows $d\left(x(k), \Sigma_{2}\right)$ versus $k$, and as can be seen from the figure, the decay is exponential. Figure 1(b) shows the input $u(k)$ versus $k$, and Fig. 1(c) shows $x_{1}(k)$ versus $k$ for $210 \leq k \leq 250$. As can be seen from these figures, the solution settles to the given periodic orbit exponentially fast.

Remark 7. Note that, as indicated in Remark 1, we have $J_{1} \neq J_{2}$ in the above case, see (50). However, again as noted in Remark 1 , the eigenvalues of both $J_{1}$ and $J_{2}$ are the same and given as -3.0101 and -0.0299 , which shows that the periodic orbit is unstable. The gain matrix $K$ given above can be found as:

$$
K=B C^{T}=\left(\begin{array}{cc}
2.2595 & 1.8651 \\
0 & 0
\end{array}\right) .
$$

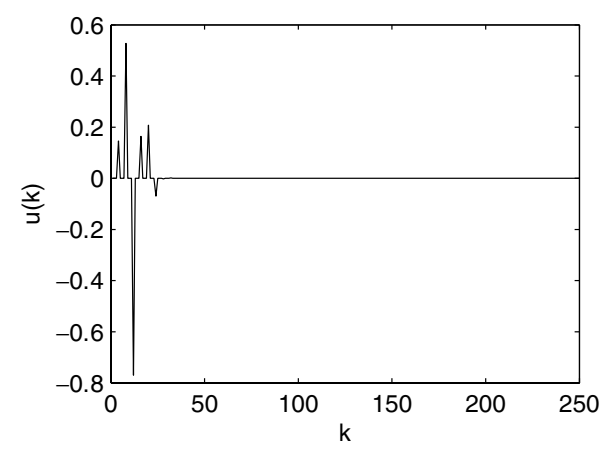

(b) 
By using (25), (51) and (56) we obtain:

$$
\begin{aligned}
& A_{1}=\left(\begin{array}{rr}
1.0028 & 0.8269 \\
-1.2150 & -1.0010
\end{array}\right), \\
& A_{2}=\left(\begin{array}{rr}
-1.2714 & -2.3454 \\
2.4918 & -1.0010
\end{array}\right) .
\end{aligned}
$$

Clearly, $A_{1} \neq A_{2}$, as noted in Remark 1. Moreover, the eigenvalues of $A_{1}$ are 0.008 and -0.007 , hence $A_{1}$ is a stable matrix; whereas the eigenvalues of $A_{2}$ are $-1.1367 \pm \jmath 2.4137$, which are different than the eigenvalues of $A_{1}$, and obviously $A_{2}$ is an unstable matrix. These results are in agreement with Remark 1.

We also simulated the same system for the case $B=\left(\begin{array}{ll}0 & 1\end{array}\right)^{T}$, i.e. the control input is only applied to (47). It can easily be shown that the pair $\left(J_{1}^{2}, B\right)$ is controllable. By following the same steps and choosing $p_{s}(\lambda)=\lambda^{2}$, we obtain

$$
C^{T}=\left(\begin{array}{ll}
2.7373 & 2.2595
\end{array}\right) \text {. }
$$

Note that in this case the controlled system becomes

$$
\begin{gathered}
x_{1}(k+1)=1+x_{2}(k)-a x_{1}(k)^{2}, \\
x_{2}(k+1)=b x_{1}(k)+u(k),
\end{gathered}
$$

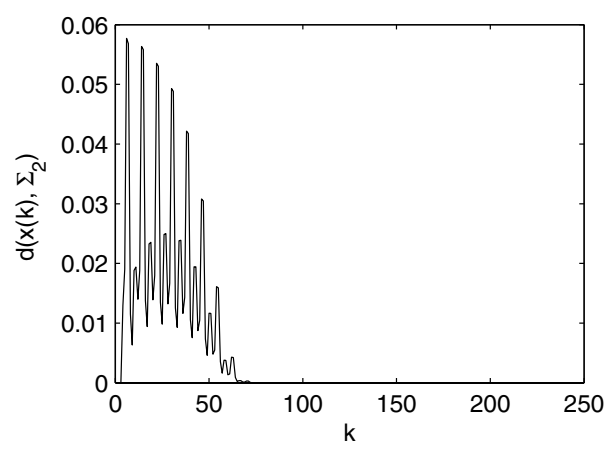

(a)

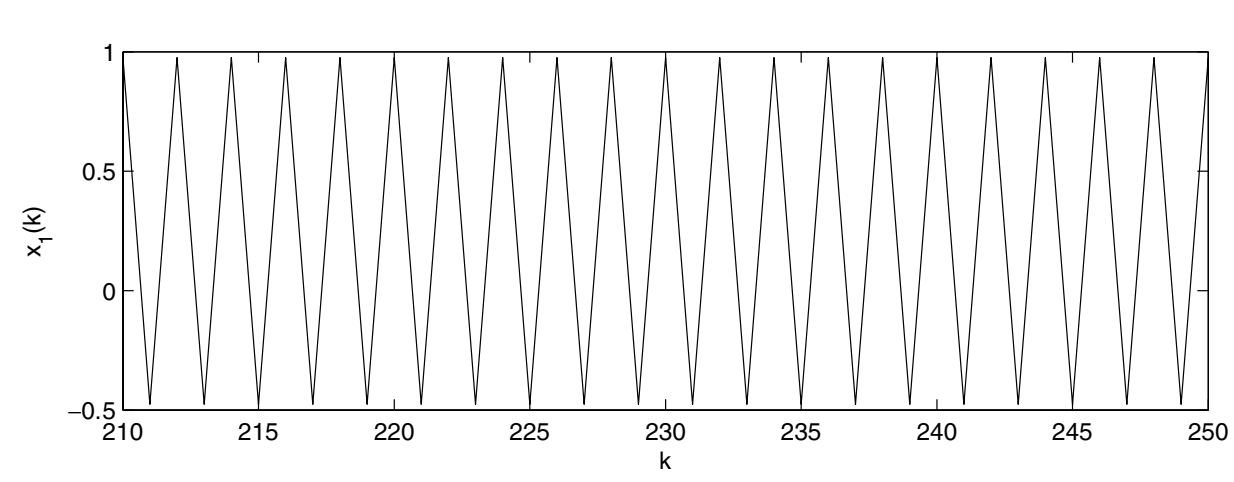

(c) where $u(k)$ is given by (44), (55) and (58), respectively. A particular simulation result for $x_{1}(1)=$ $-0.47, x_{2}(1)=0.29$ and $\epsilon_{m}=0.6$ is given in Fig. 2 . Figure $2(\mathrm{a})$ shows $d\left(x(k), \Sigma_{2}\right)$ versus $k$, and as can be seen from the figure, the decay is exponential. Figure 2(b) shows the input $u(k)$ versus $k$, and Fig. 2(c) shows $x_{1}(k)$ versus $k$ for $210 \leq k \leq 250$. As can be seen from these figures, the solution settles to the given periodic orbit exponentially fast.

For the second case of simulations, we considered the coupled map lattices by using the tent map. The model we use is given by the following:

$$
\begin{aligned}
& x_{1}(k+1)=f\left(x_{1}(k)\right)+\epsilon\left(f\left(x_{2}(k)\right)-f\left(x_{1}(k)\right)\right), \\
& x_{2}(k+1)=f\left(x_{2}(k)\right)+\epsilon\left(f\left(x_{1}(k)\right)-f\left(x_{2}(k)\right)\right),
\end{aligned}
$$

where $\epsilon>0$ is a coupling constant and the tent map is given by:

$$
f(x)=\left\{\begin{array}{ll}
\mu x & 0 \leq x<0.5 \\
\mu(1-x) & 0.5 \leq x \leq 1
\end{array} .\right.
$$

For the values $\mu=1.9$ and $\epsilon=0.1$, the system given above exhibits chaotic behavior, and in

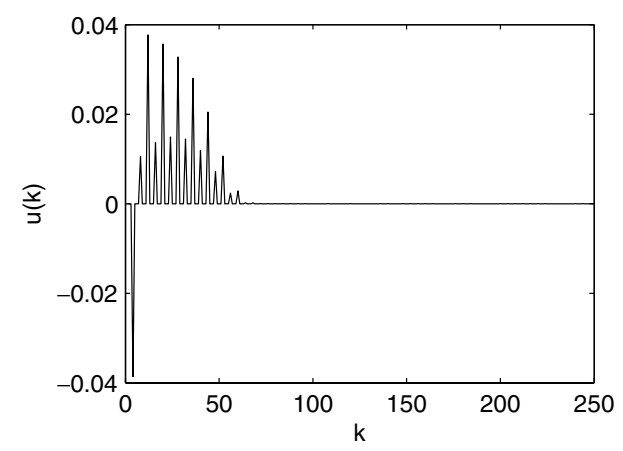

(b)

Fig. 2. DPDFC applied to Hénon map, $B=\left(\begin{array}{ll}0 & 1\end{array}\right)^{T}$, (a) $d\left(x(k), \Sigma_{2}\right)$ versus $k$, (b) $u(k)$ versus $k$, (c) $x_{1}(k)$ versus $k$ for $210 \leq k \leq 250$. 
particular it possesses a period 3 unstable orbit $\Sigma_{3}=\left\{w_{1}^{*}, w_{2}^{*}, w_{3}^{*}\right\}$ given as:

$$
\begin{gathered}
w_{1}^{*}=\left(\begin{array}{l}
0.244670240 \\
0.630468494
\end{array}\right), \quad w_{2}^{*}=\left(\begin{array}{l}
0.488597096 \\
0.678386219
\end{array}\right), \\
w_{3}^{*}=\left(\begin{array}{l}
0.896607653 \\
0.642793012
\end{array}\right) .
\end{gathered}
$$

First, we assume that $C$ is given as $C^{T}=\left(\begin{array}{ll}1 & 0\end{array}\right)$, i.e. only the first variable is measurable. By following the steps given in the previous section, using $p_{s}(\lambda)=\lambda^{2}$ and (42), we obtain $B=$ $(6.536619 .2487)^{T}$. Note that in this case DPDFC scheme for the system given by (61) and (62) becomes:

$$
\begin{aligned}
x_{1}(k+1)= & f\left(x_{1}(k)\right)+\epsilon\left(f\left(x_{2}(k)\right)-f\left(x_{1}(k)\right)\right) \\
& +6.5366 u(k), \\
x_{2}(k+1)= & f\left(x_{2}(k)\right)+\epsilon\left(f\left(x_{1}(k)\right)-f\left(x_{2}(k)\right)\right) \\
& +19.2487 u(k),
\end{aligned}
$$

where $u(k)$ is given by (44) and (55) and $C^{T}=(10)$. It can also be easily shown that the observability

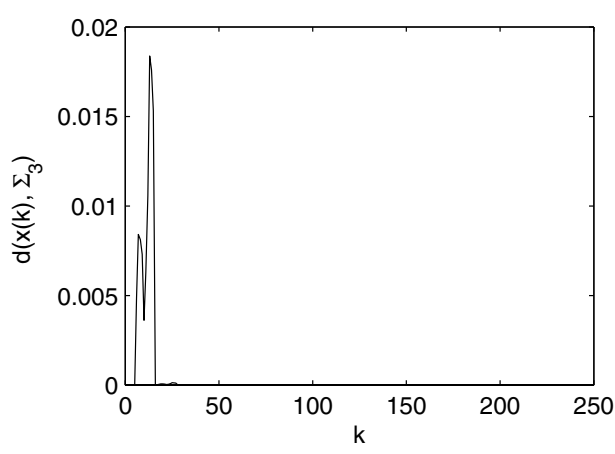

(a) condition mentioned in Theorem 3 is satisfied in this case. A particular simulation result for $x_{1}(1)=$ 0.2446, $x_{2}(1)=0.63$ and $\epsilon_{m}=0.6$ is given in Fig. 3 . Figure 3(a) shows $d\left(x(k), \Sigma_{3}\right)$ versus $k$, and as can be seen from the figure, the decay is exponential. Figure $3(\mathrm{~b})$ shows the input $u(k)$ versus $k$, and Fig. 3(c) shows $x_{1}(k)$ versus $k$ for $210 \leq k \leq 250$. As can be seen from these figures, the solution settles to the given periodic orbit exponentially fast.

Finally, we consider the case $C^{T}=\left(\begin{array}{ll}0 & 1\end{array}\right)$, i.e. only the second variable is measurable. By following the steps given in the previous section, using $p_{s}(\lambda)=\lambda^{2}$ and (42), we obtain $B=$ $(19.24876 .5366)^{T}$. It can also be easily shown that the observability condition mentioned in Theorem 3 is satisfied in this case. Note that in this case DPDFC scheme for the system given by (61) and (62) becomes:

$$
\begin{aligned}
x_{1}(k+1)= & f\left(x_{1}(k)\right)+\epsilon\left(f\left(x_{2}(k)\right)-f\left(x_{1}(k)\right)\right) \\
& +19.2487 u(k), \\
x_{2}(k+1)= & f\left(x_{2}(k)\right)+\epsilon\left(f\left(x_{1}(k)\right)-f\left(x_{2}(k)\right)\right) \\
& +6.5366 u(k),
\end{aligned}
$$

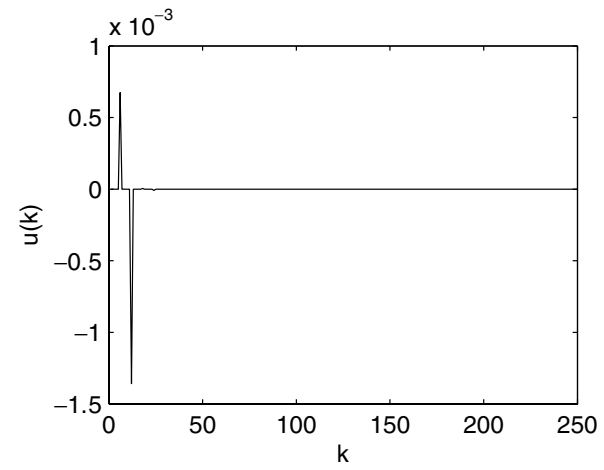

(b)

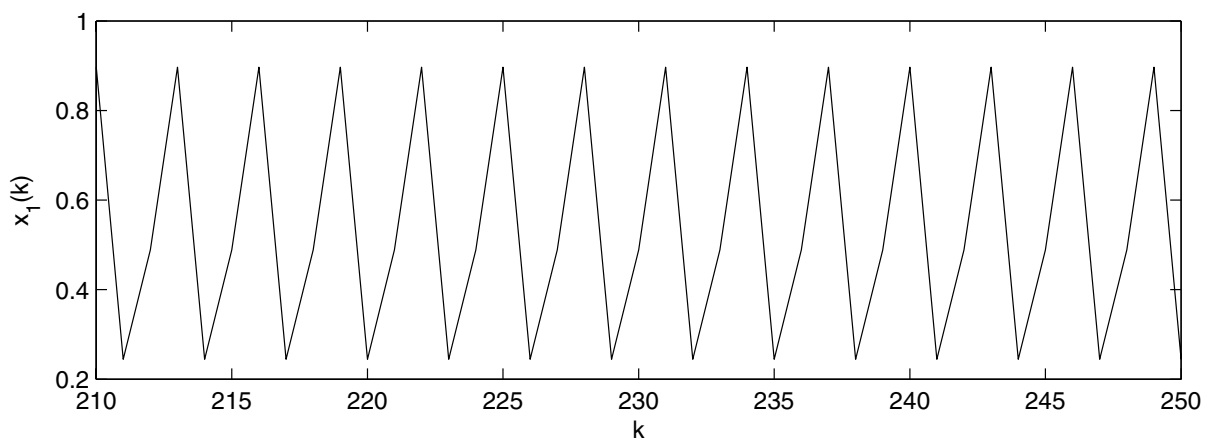

(c)

Fig. 3. DPDFC scheme applied to coupled tent map, $C=(10)^{T}$, (a) $d\left(x(k), \Sigma_{3}\right)$ versus $k$, (b) $u(k)$ versus $k$, (c) $x_{1}(k)$ versus $k$ for $210 \leq k \leq 250$. 


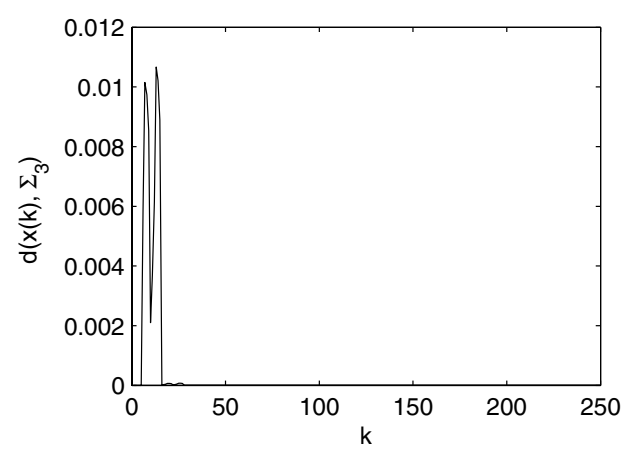

(a)

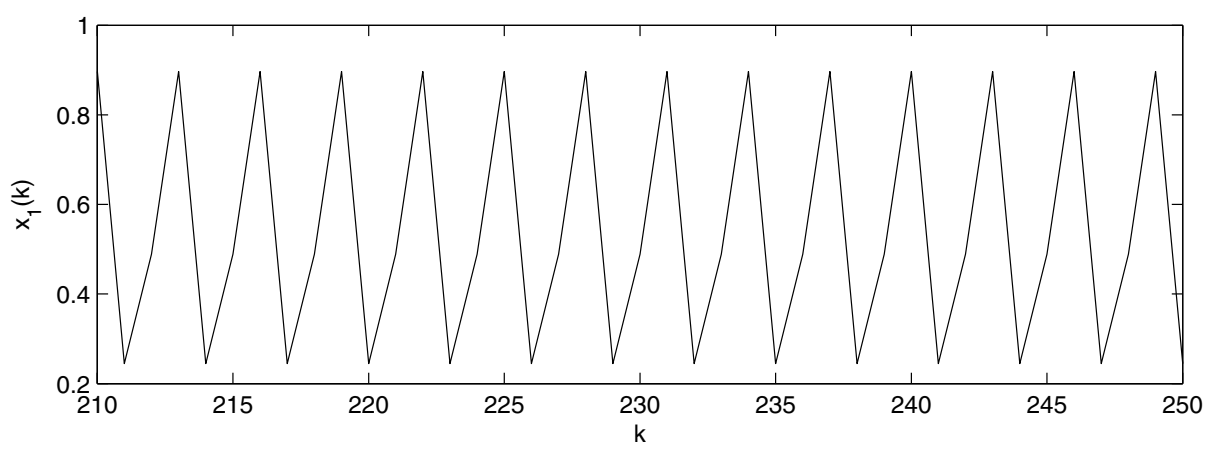

(c)

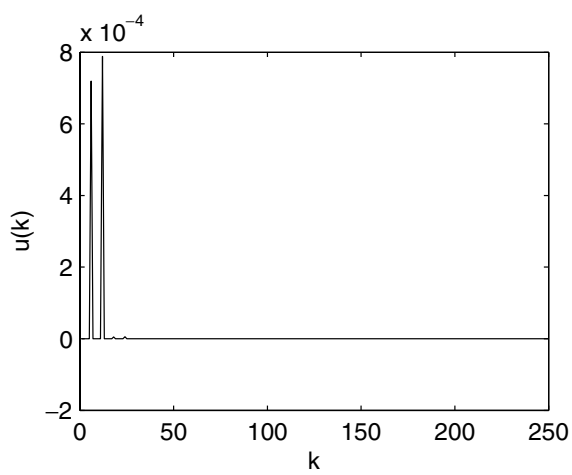

(b)

Fig. 4. DPDFC scheme applied to coupled tent map, $C=\left(\begin{array}{ll}0 & 1\end{array}\right)^{T}$, (a) $d\left(x(k), \Sigma_{3}\right)$ versus $k$, (b) $u(k)$ versus $k$, (c) $x_{1}(k)$ versus $k$ for $210 \leq k \leq 250$.

where $u(k)$ is given by (44) and (55) and $C^{T}=$ (0 1). A particular simulation result for $x_{1}(1)=$ $0.2441, x_{2}(1)=0.6304$ and $\epsilon_{m}=0.6$ is given in Fig. 4. Figure $4(\mathrm{a})$ shows $d\left(x(k), \Sigma_{3}\right)$ versus $k$, and as can be seen from the figure, the decay is exponential. Figure $4(\mathrm{~b})$ shows the input $u(k)$ versus $k$, and Fig. 4 (c) shows $x_{1}(k)$ versus $k$ for $210 \leq k \leq 250$. As can be seen from these figures, the solution settles to the given periodic orbit exponentially fast.

\section{Conclusion}

In this work we study a problem related to the stabilization of unstable periodic orbits of chaotic systems. We assumed that the control input is scalar and assumed that either a vector related to the control input $(B)$, or to the measurement $(C)$ is given. Then the problem is to find an appropriate control gain matrix $K=B C^{T}$ along with a (scalar) control input $u$ so that the given unstable periodic orbit becomes stable. The solution we propose is a modification of Delayed Feedback Control (DFC) and is called as Double Period DFC, see [Morgül, 2006]. We showed that under certain conditions the proposed scheme achieves local exponential stabilization of the given unstable periodic orbit. Note that in DPDFC, any hyperbolic periodic orbit can be stabilized with an appropriate gain $K$, see [Morgül, 2006]. A similar result was obtained in [Morgül, 2005b] by using yet another modification of DFC scheme. While in [Morgül, 2005b, 2006], the gain matrix $K$ is arbitrary, hence the control input $u$ is not a scalar signal in general, in the present work we put a restriction on the gain $K$ and assumed that it has the form $K=B C^{T}$, where either $B$ or $C$ is a given vector. As a result, the required control input becomes a scalar signal. We showed that the proposed scheme achieves stabilization when the given periodic orbit is hyperbolic, and moreover the controlled system satisfies either a controllability condition when $B$ is given, or an observability condition when $C$ is given. Since these conditions are generic (i.e. are almost always satisfied), it can be stated that almost any unstable periodic orbit can be stabilized with the proposed scheme by a scalar control input. 


\section{References}

Alligood, K. T., Sauer, T. D. \& Yorke, J. A. [1997] Chaos: An Introduction to Dynamical Systems (SpringerVerlag, NY).

Bleich, M. E. \& Socolar, J. E. S. [1996] "Stability of periodic orbits controlled by time delay feedback," Phys. Lett. A 210, 87-94.

Chen, G. \& Dong, X. [1998] From Chaos to Order: Methodologies, Perspectives and Applications (World Scientific, Singapore).

Devaney, R. L. [1987] Chaotic Dynamical Systems (Addison-Wesley, Redwood City).

Fradkov, A. L. \& Pogromsky, A. Y. [1998] Introduction to Control of Oscillations and Chaos (World Scientific, Singapore).

Hino, T., Yamamoto, S. \& Ushio, T. [2002] "Stabilization of unstable periodic orbits of chaotic discrete time systems using prediction based feedback control," Int. J. Bifurcation and Chaos 12, 439446.

Kailath, T. [1980] Linear Systems (Prentice-Hall, NJ).

Khalil, H. K. [2002] Nonlinear Systems, 3rd edition (Prentice-Hall, Upper Saddle River).

Kittel, A., Parisi, J. \& Pyragas, K. [1995] "Delayed feedback control of chaos by self-adapted delay time," Phys. Lett. A 198, 433-436.

Morgül, Ö. \& Solak, E. [1996] "Observer based synchronization of chaotic systems," Phys. Rev. E 54, 48034811.

Morgül, Ö. \& Solak, E. [1997] "On the synchronization of chaotic systems by using state observers," Int. J. Bifurcation and Chaos 7, 1307-1322.

Morgül, Ö. [2003a] "On the stability of delayed feedback controllers," Phys. Lett. A 314, 278-285.
Morgül, Ö. [2003b] "A model based scheme for anticontrol of some chaotic systems," Int. J. Bifurcation and Chaos 13, 3449-3457.

Morgül, Ö. [2005a] "On the stability of delayed feedback controllers for discrete time systems," Phys. Lett. A 335, 31-42.

Morgül, Ö. [2005b] "On the stabilization of periodic orbits for discrete time chaotic systems," Phys. Lett. A 335, 127-138.

Morgül, Ö. [2006] "Stabilization of unstable periodic orbits for discrete time chaotic systems by using periodic feedback," Int. J. Bifurcation and Chaos 16,311323.

Nakajima, H. \& Ueda, Y. [1998] "Half period delayed feedback control for dynamical systems with symmetries," Phys. Rev. E 55, 1757-1763.

Ott, E., Grebogi, C. \& Yorke, J. A. [1990] "Controlling chaos," Phys. Rev. Lett. 64, 1196-1199.

Pyragas, K. [1992] "Continuous control of chaos by self controlling feedback," Phys. Lett. A 170, 421-428.

Pyragas, K. [1995] "Control of chaos via extended delay feedback," Phys. Lett. A 206, 323-330.

Schuster, H. G. \& Stemmler, M. B. [1997] "Control of chaos by oscillating feedback," Phys. Rev. E 56, 64106417.

Socolar, J. E., Sukow, D. W. \& Gauthier, D. J. [1994] "Stabilizing unstable periodic orbits in fast dynamical systems," Phys. Rev. E 50, 3245-3248.

Solak, E., Morgül, Ö. \& Ersoy, U. [2001] "Observer based control of a class of chaotic systems," Phys. Lett. A 279, $47-55$.

Ushio, T. [1996] "Limitation of delayed feedback control in nonlinear discrete time systems," IEEE Trans. Circuits Syst.-I 43, 815-816. 